\title{
Ärztliche Qualitätsarbeit - machen Sie sich ein Bild!
}

\section{Christoph Bosshard}

Vizepräsident der FMH, Departementsverantwortlicher Daten, Demographie und Qualität

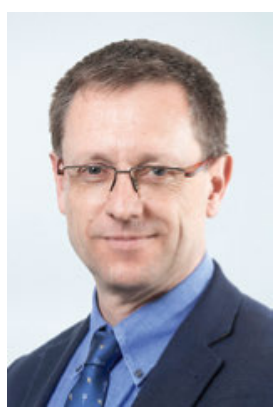

First they ignore you, then they laugh at you, then they fight you, then you win.

Mahatma Gandhi

Dieser Ausspruch, welcher Gandhi zugeschrieben wird, kommt mir in letzter Zeit immer wieder in den Sinn, wenn ich Äusserungen zur ärztlichen Qualitätsarbeit seitens Politik, Verwaltung und einiger Versicherer vernehme. Die Tatsache, dass unsere Aktivitäten in der Qualitätssicherung und -entwicklung systematisch skotomisiert werden, verlangt nach noch mehr Kommunikation. Ganz nach dem Motto "Tue Gutes und sprich darüber» werden wir seitens Ärzteschaft noch intensiver über unsere Aktivitäten informieren als bisher.

Dass die FMH seit Jahrzehnten die Transparenz hoch hält, belegt zum Beispiel die seit 1940 veröffentlichte Ärztestatistik wie auch die seit den 70er Jahren bis zum Bundesveto 2013 publizierte Einkommensstatistik. Nun lösen wir die mit der Gründung der Schweizerischen Akademie für Qualität in der Medizin (SAQM) im Jahr 2012 abgegebenen Versprechen ein und zeigen im Porträt-Artikel auf, wo wir gut fünf Jahre später stehen. Die diversen Projekte und Aktivitäten haben bisweilen Pionier-Charakter, zeichnen sich auch durch international anerkannte Innovation aus und werden mit grossem Engagement und Aufwand durch die beteiligten Organisationen mitgetragen.

\section{Das Knowhow und Engagement von Mit-} gliedern und Ärzteorganisationen macht erst innovative Projekte möglich.

Das zugrunde liegende Knowhow und die oft unentgeltlich durchgeführten Arbeiten sind für Aussenstehende oft nicht sichtbar, was zur eingangs erwähnten
Kommunikationsherausforderung passt. Wenn die gesamten Aufwendungen im Qualitätsbereich erfasst werden wollen, um schliesslich in eine korrekte Aufwand-Nutzen-Abwägung Eingang zu finden, dürfen nicht nur Geldflüsse betrachtet werden. Auf der Nutzen-Seite ist ebenfalls eine Analyse notwendig, welche insbesondere die gesamtwirtschaftliche Sichtweise berücksichtigen muss, um neue Fehlanreize zu vermeiden.

In diesem Kontext sind auch die administrativen Aufwendungen zu diskutieren. Die über Jahre hinweg kontinuierlich gestiegene Belastung der ärztlichen Berufstätigkeit durch administrative Aufgaben führt dazu, dass Ärztinnen und Ärzte stets mehr Zeit am Papier oder Computer aufwenden müssen, anstatt dass sie auch diese für ihre Kernaufgaben - die Behandlung der

Unsere Patienten und ihre Bedürfnisse müssen bei allen Qualitätsarbeiten im Vordergrund stehen.

Patienten - einsetzen können. Liegt nicht hier eines der wesentlichen Qualitätsprobleme? Die Qualitätsarbeiten müssten also letztlich unter anderem dazu führen, dass unsere Patientinnen und Patienten mit ihren Bedürfnissen und Anliegen im Vordergrund stehen, und nicht Röntgenbilder, Laborwerte, Arbeitsanweisungen oder andere Blau-, Weiss- oder SchwarzBücher. Auch hierzu existiert ein Projekt der SAQM. Ich lade Sie nun gerne ein, den Artikel zur SAQM ab Seite 630 sorgfältig zu studieren und auch auf unserer Homepage www.saqm.ch zu stöbern. All unseren Organisationen und Mitgliedern, welche unermüdlich bei diesen Thematiken mitgearbeitet haben und immer noch mitarbeiten, danke ich herzlich. Sie sind es nämlich, welche die SAQM ausmachen! 